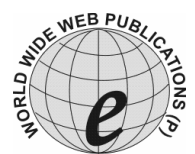

E= $=$, (C)

http://WWW.e-journals.net

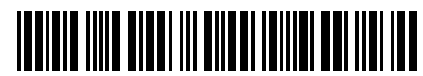

ISSN: 0973-4945; CODEN ECJHAO

E-Journal of Chemistry

2012, 9(1), 219-223

\title{
Synthesis of Novel 6-Cyano-9-(aryl)-9H-purine Derivatives Via Formamidine Intermediates
}

\author{
HASHEMI, SEYYED MILAD ${ }^{\S *}$, YAHYAZADEH, ASIEH ${ }^{\S}$ and NAMI, NAVABEH \\ ${ }^{\S}$ Department of Chemistry, Guilan University \\ P.O. Box 1914, Rasht, Guilan, Iran \\ Department of Chemistry, Islamic Azad University \\ Qaemshahr, Mazandaran, Iran \\ seyyedmiladhashemi@hotmail.com
}

Received 19 August 2010; Accepted 20 October 2010

\begin{abstract}
Novel 9-substituted cyanopurine derivatives (4a-d) were synthesized in three steps in high yield. Diaminomaleonitrile (1) reacted with triethyl orthoformate to afford (Z)- $N$-[2-amino-1,2-dicyanovinyl] formimidate (2) which was converted to aryl-(Z)- $N$-[2-amino-1,2-dicyanovinyl] formamidines (3a-d) in the presence of a catalytic amount of anilinium chloride and aromatic amines in ethanol at room temperature under inert atmosphere (Argon). Furthermore, the reaction of (3a-d) with triethyl orthoformate afforded novel 6-cyano-9(aryl)-9H-purine derivatives (4a-d) which can be used without further purification. All compounds have been fully characterized by spectroscopic data.
\end{abstract}

Keywords: Formimidate, Aromatic amines, Formamidine, Triethyl orthoformate, 6-Cyanopurine

\section{Introduction}

Amidines are an important class of compounds which have proved to be intermediates in a number of biological processes ${ }^{1-3}$. The chemistry and preparation of amidines have been reviewed by Shriner and Neumann ${ }^{4}$. Purine, purine nucleosides and their analogues have been extremely useful as an anti-cancer agents ${ }^{5-8}$. Robines, in 1964, reviewed the anti-tumour activity of purine and purine nucleosides from a structure-activity stand-point. Subsequently several reviews on this subject have been published ${ }^{9-12}$. Since the discovery of acyclovir great research effort has been devoted to the synthesis of new acyclic nucleoside analogues as potential anti-herpes (HSV) and anti-human cytomegalovirus (HCMV) agents ${ }^{13}$. The chemotherapeutic uses of purines and purine analogues have prompted tremendous efforts towards their synthesis, both in academia and in the pharmaceutical industry. 6 -cyanopurines are usually obtained by cyanide ion substitution of either 6 -iodo ${ }^{14}$, 6-tosyl- ${ }^{15}$, 
6-methyle-sulfonyl ${ }^{16,17}$ and 6-trimethylammonio ${ }^{18}$ purine derivatives, or by dehydration of the 6-oximo derivatives with acetic anhydride ${ }^{19}$. The yields from these reactions are only moderate to poor. It has previously reported ${ }^{20}$ that 6 -cyanopurines can be obtained in good yields by reaction of 1-substituted-5-amino-4-(cyanoformimidoyl)-imidazoles with carboxylic acid anhydrides, but it is not always easy to isolate and handle these imidazole intermediates and we were interested in developing a simpler route to 6-cyanopurines. In continuation of our work on the synthesis of heterocyclic systems containing nitrogen ${ }^{21-26}$, we described here the synthesis of new 6-cyano-9-(aryl)-9H-purine derivatives.

\section{Experimental}

All solvents purified and dried using estabilished procedures. The ${ }^{1} \mathrm{H}$ NMR spectra were recorded on Bruker XL $500(500 \mathrm{MHz})$ instruments, IR spectra on a shimadzu IR-470 spectrophotometer and mass spectra were recorded on a Kratos concept instrument. The $\mathrm{C}, \mathrm{H}, \mathrm{N}$ analyses were performed by the micro analytical service of the N.I.O.C. Research Institute of Petroleum Industry. The melting points were measured on an Electrothermal digital melting point apparatus and are uncorrected.

General procedure for the preparation of the aryl-(Z)-N-[2-amino-1,2dicyanovinyl]formamidines (3a-d)

The aromatic amine $(1.22 \mathrm{mmol})$ was added to a suspension of formimidate (2) $(0.20 \mathrm{~g}$, $1.22 \mathrm{mmol})$ that previously was produced ${ }^{27}$, in dry ethanol or ethylacetate $(6 \mathrm{~mL})$, which contained anilinium chloride $(0.01 \mathrm{~g})$. The mixture was stirred at room temperature under inert atmosphere (argon) for 9 hours. The reaction followed by TLC $(8: 2$ ethylacetate / petroleumether) until all the formimidate (2) had disappeared and the amidine was isolated by filtration. The most cases, the product was pale green to white. The precipitate was washed with diethylether and air-dried to give the analytically pure product.

\section{4-Ethoxyphenyl-(Z)-N-[2-amino-1,2-dicyanovinyl]formamidine (3a)}

(92.80\%), Green, mp 148-150 ${ }^{\circ} \mathrm{C}$; MS: $m / z 255$ (M) ${ }^{+}$; IR (KBr): 3450, 3350, 1470, 3100, 2950, 2210, 1630, 1590, 1375, 1465, 1280, 1240, $830 \mathrm{~cm}^{-1} ;{ }^{1} \mathrm{H}$ NMR (DMSO-d 6 ) $\delta 9.83$ (br.s, $1 \mathrm{H}, \mathrm{NH}), 7.70$ (d, $J=88 \mathrm{~Hz}, 2 \mathrm{H}, \mathrm{Ar}-\mathrm{H}), 6.87$ (d, $J=8 \mathrm{~Hz}, 2 \mathrm{H}, \mathrm{Ar}-\mathrm{H}), 6.37$ (br.d, 2H, $\left.\mathrm{NH}_{2}\right), 4.00\left(\mathrm{q}, J=13.90 \mathrm{~Hz}, 2 \mathrm{H}, \mathrm{CH}_{2}\right), 3.35(\mathrm{~s}, 1 \mathrm{H}, \mathrm{CH}), 1.32\left(\mathrm{t}, J=13.90 \mathrm{~Hz}, 3 \mathrm{H}, \mathrm{CH}_{3}\right)$. Anal. Calcd. for $\mathrm{C}_{13} \mathrm{H}_{13} \mathrm{~N}_{5} \mathrm{O}$ : C, 61.17; H, 5.10; N, 27.39. Found: C, 61.20; H, 6.56; N, 8.15.

\section{1,4-Diaminophenyl-bis[(Z)-9N,9N'-(2-amino-1,2-dicyanovinyl)]formamidine (3b)}

(71.51\%), Green, $\mathrm{mp}>340{ }^{\circ} \mathrm{C}$; MS: $m / z 344(\mathrm{M})^{+}$; IR (KBr): 3450, 3350, 3200, 2950, 2200, 1640, 1600, 1585, 1500, 1300, 1640, 810, $780 \mathrm{~cm}^{-1} ;{ }^{1} \mathrm{H}$ NMR (DMSO-d $\left.{ }_{6}\right) \delta 9.97$ (br.s, 1H, NH), 7.73 (s, 4H, Ar-H), 6.55 (d, $J=8.69 \mathrm{~Hz}, 1 \mathrm{H}, \mathrm{CH}), 6.32$ (br.s, $2 \mathrm{H}, \mathrm{NH}_{2}$ ). Anal. Calcd. for $\mathrm{C}_{16} \mathrm{H}_{12} \mathrm{~N}_{2}$ : C, 55.81; H, 3.48; N, 40.69. Found: C, 55.70; H, 3.50; N, 40.58.

\section{2-Methoxybenzyl-(Z)- $N$-[2-amino-1,2-dicyanovinyl]formamidine (3c)}

(65.22\%), White, mp 147-150 ${ }^{\circ} \mathrm{C}$; MS: $m / z 344$ (M) ${ }^{+}$; IR (KBr): 3500, 3400, 3100, 2950, 2200, 1620, 1550, 1480, 1300, 1465, 1340, 850, $830 \mathrm{~cm}^{-1} ;{ }^{1} \mathrm{H}$ NMR (DMSO-d ${ }_{6}$ ) $\delta 7.97$ (br.d, $J=4.90 \mathrm{~Hz}, 1 \mathrm{H}, \mathrm{NH}), 7.71(\mathrm{~d}, J=4.04 \mathrm{~Hz}, 1 \mathrm{H}, \mathrm{CH}), 7.27(\mathrm{q}, J=7.52 \mathrm{~Hz}, 2 \mathrm{H}, \mathrm{Ar}-\mathrm{H}), 7.00(\mathrm{~d}$, $J=7.94 \mathrm{~Hz}, 1 \mathrm{H}, \mathrm{Ar}-\mathrm{H}), 6.91(\mathrm{t}, \mathrm{J}=7.43 \mathrm{~Hz}, 1 \mathrm{H}, \mathrm{Ar}-\mathrm{H}), 6.06\left(\mathrm{~s}, 2 \mathrm{H}, \mathrm{NH}_{2}\right), 4.47(\mathrm{~d}, 2 \mathrm{H}$, $\mathrm{CH}_{2}$ ), 3.81 (s, $3 \mathrm{H}, \mathrm{OCH}_{3}$ ). Anal. Calcd. for $\mathrm{C}_{13} \mathrm{H}_{13} \mathrm{~N}_{5} \mathrm{O}: \mathrm{C}, 61.17 ; \mathrm{H}, 5.09 ; \mathrm{N}, 27.45$. Found: C, 61.20; H, 5.10; N, 27.39. 


\section{4-Hydroxy-(Z)-N-[2-amino-1,2-dicyanovinyl]formamidine (3d)}

(94\%), Pale green, $\mathrm{mp} 130{ }^{\circ} \mathrm{C}$; MS: $m / z 227(\mathrm{M})^{+}$; IR (KBr): 3450, 3325, 3200, 3100, $2900,2400,1645,1590,1570,1510,1420,1360,1320,960,820,750 \mathrm{~cm}^{-1} ;{ }^{1} \mathrm{H}$ NMR $\left(\mathrm{DMSO}_{6}\right) \delta 9.72(\mathrm{~s}, 1 \mathrm{H}, \mathrm{NH}), 9.22(\mathrm{~s}, 1 \mathrm{H}, \mathrm{CH}), 7.64(\mathrm{~d}, 2 \mathrm{H}, \mathrm{Ar}-\mathrm{H}), 6.72(\mathrm{~s}, 2 \mathrm{H}, \mathrm{Ar}-$ $\mathrm{H}), 6.28\left(\mathrm{~s}, 2 \mathrm{H}, \mathrm{NH}_{2}\right)$. Anal. Calcd. for $\mathrm{C}_{11} \mathrm{H}_{9} \mathrm{~N}_{5} \mathrm{O}: \mathrm{C}, 58.14 ; \mathrm{H}, 3.96 ; \mathrm{N}, 30.83$. Found: C, 57.98; H, 4.01; N, 30.39.

\section{General procedure for the preparation of the 6-cyano-9-(aryl)-9H-purine $(\mathbf{4 a - d})$}

The mixture of formamidine (3a-d) $(0.40 \mathrm{mmol})$ and triethyl orthoformate $(3 \mathrm{~mL}$, $6.33 \mathrm{mmol})$ were heated at reflux temperature for 6 hours until TLC (8:2, ethylacetate / petroleum ether) showed that all the formamidine (3a-d) had disappeared and the 9-substituted cyanopurines (3a-d) was isolated by filtration. The most cases the product was grey. The precipitate was washed with dry petroleum ether and air-dried. Obtained precipitates were recrystallized by ethanol to give the analytically pure product.

\section{6-Cyano-9-(4-ethoxyphenyl)-9H-purine (4a)}

(70\%), Pale gray, mp 182-184 ${ }^{\circ} \mathrm{C}$; MS : $m / z 265(\mathrm{M})^{+}$; IR (KBr): 3100, 2900, 2380, 1590, 1475, 1510, 1340, 1440, $1350 \mathrm{~cm}^{-1} ;{ }^{1} \mathrm{H}$ NMR (DMSO-d 6 ) $\delta 9.18$ (s, 1H, H (Pyrimidine)), 9.09 (s, 1H, H (Imidazole)), 7.69 (d, $J=8.89 \mathrm{~Hz}, 2 \mathrm{H}, \mathrm{Ar}-\mathrm{H}), 7.10(\mathrm{~d}, J=8.92 \mathrm{~Hz}, 2 \mathrm{H}, \mathrm{Ar}-\mathrm{H})$, $4.07\left(\mathrm{q}, J=6.93 \mathrm{~Hz}, 2 \mathrm{H}, \mathrm{CH}_{2}\right), 1.33\left(\mathrm{t}, J=6.93 \mathrm{~Hz}, 3 \mathrm{H}, \mathrm{CH}_{3}\right)$. Anal. Calcd. for $\mathrm{C}_{14} \mathrm{H}_{11} \mathrm{~N}_{5} \mathrm{O}$ : C, 63.39; H, 4.15; N, 26.41. Found:C, 62.40; H, 4.16; N, 26.36.

\section{1,4-Phenylen-bis[9N,9N'-6-cyano-9H]purine (4b)}

(71.1\%), green, $\mathrm{mp}>340{ }^{\circ} \mathrm{C}$; MS : $m / 2364.40(\mathrm{M})^{+}$; IR (KBr): 3100, 2350, 1510, 1590, 1400, $1350 \mathrm{~cm}^{-1} ;{ }^{1} \mathrm{H}$ NMR (DMSO-d $\left.{ }_{6}\right) \delta 9.41(\mathrm{~s}, 2 \mathrm{H}, \mathrm{H}$ (Pyrimidine)), $9.20(\mathrm{~s}, 2 \mathrm{H}, \mathrm{H}$ (Imidazole)), 8.19 (s, 4H, Ar-H). Anal. Calcd. for $\mathrm{C}_{18} \mathrm{H}_{8} \mathrm{~N}_{10}$ : C, 59.34; H, 2.23; N, 47.45. Found: C, 60.02; H, 2.23; N, 47.45.

\section{6-Cyano-9-(2-methoxybenzyl)-9H-purine (4c)}

(80.40\%), Gray, mp 147-150 ${ }^{\circ} \mathrm{C}$; MS : $m / 2293(\mathrm{M})^{+}$; IR (KBr): 3100, 2900, 2380, 1590, 1485, 1460, 1400, 1320, 1440, $1380 \mathrm{~cm}^{-1} ;{ }^{1} \mathrm{H}$ NMR $\left(\mathrm{CDCl}_{3}-\mathrm{d}_{1}\right) \delta 9.06(\mathrm{~s}, 1 \mathrm{H}, \mathrm{H}$ (Pyrimidine)), 8.35 (s, 1H, H (Imidazole)), 7.43 (d, $J=7.38 \mathrm{~Hz}, 1 \mathrm{H}, \mathrm{Ar}-\mathrm{H}), 7.35$ (t, $J=7.94$ $\mathrm{Hz}, 1 \mathrm{H}, \mathrm{Ar}-\mathrm{H}), 6.97(\mathrm{t}, J=7.47 \mathrm{~Hz}, 1 \mathrm{H}, \mathrm{Ar}-\mathrm{H}), 6.92(\mathrm{~d}, J=8.28 \mathrm{~Hz}, 1 \mathrm{H}, \mathrm{Ar}-\mathrm{H}), 5.46(\mathrm{~s}, 2 \mathrm{H}$, $\mathrm{CH}_{2}$ ), 3.87 (s, $3 \mathrm{H}, \mathrm{OCH}_{3}$ ). Anal. Calcd. for $\mathrm{C}_{14} \mathrm{H}_{11} \mathrm{~N}_{5} \mathrm{O}: \mathrm{C}, 57.33 ; \mathrm{H}, 3.75 ; \mathrm{N}, 23.89$. Found: C, 56.08; H, 3.70; N, 24.07.

\section{6-Cyano-9-(4-hydroxy)-9H-purine (4d)}

(75\%), Brown, mp 208-210 ${ }^{\circ} \mathrm{C}$; MS: $m / 2237(\mathrm{M})^{+}$; IR (KBr): 3200, 2380, 1500, 1460, 1400, $1320 \mathrm{~cm}^{-1}$; ${ }^{1} \mathrm{H}$ NMR (DMSO-d 6 ) $\delta 10.00(\mathrm{~s}, 1 \mathrm{H}, \mathrm{OH}), 9.15$ (s, 1H, H (Pyrimidine)), 9.11 (s, 1H, H (Imidazole)), 7.59 (d, J = 8.75 Hz, 2H, Ar-H), $6.95(\mathrm{~d}, J=8.79 \mathrm{~Hz}, 2 \mathrm{H}, \mathrm{Ar}-\mathrm{H})$. Anal. Calcd. for $\mathrm{C}_{12} \mathrm{H}_{7} \mathrm{~N}_{5} \mathrm{O}: \mathrm{C}, 60.75 ; \mathrm{H}, 2.95 ; \mathrm{N}, 29.53$. Found: C, 59.90; H, 2.99; N, 29.95.

\section{Results and Discussion}

Formimidate (2) was prepared in high yield from diaminomaleonitrile (1) and triethyl orthofarmate, according to a previously described procedure ${ }^{27}$. Having obtained the imidate (2) in good yield it was then treated with aryl or benzylamine in a 1:1 or 1:2 molar ratio in dry ethanol in the presence of a catalytic amount of anilinium chloride and argon atmospheres. These compounds would be important intermediates 
for the synthesis of a range of 9-arylpurines and 9-aryl-1,2-dihydropurines. We attempted to cyclise these amidines (3a-d) to obtain compounds (4a-d), via refluxing them with triethyl orthofarmate (Scheme 1). The reactions followed by TLC (8:2 ethylacetate / petroleum ether) to all the starting material had been consumed. These compounds filtered, washed with petroleum ether and diethylether, recrystallized with ethanol. These were fully characterized by IR, ${ }^{1} \mathrm{H}-\mathrm{NMR}$, MS spectroscopy and elemental analysis.

The elemental analysis results of amidines (3a-d) were satisfactory. The infrared spectrum of amidines (3a-d) confirmed the presence of the $\mathrm{NH}$ and $\mathrm{C}=\mathrm{N}$ stretching vibrations in 34803120 and $1600-1640 \mathrm{~cm}^{-1}$ respectively. The infrared spectrum also showed a sharp absorption band at 2220-2200 $\mathrm{cm}^{-1}$ for the CN stretching vibration. The mass spectra showed peaks at $\mathrm{m} / \mathrm{z}$ 255 (3a), 344 (3b), 255 (3c) and 227 (3d) respectively.

The ${ }^{1} \mathrm{H}$ NMR spectra of compounds $(\mathbf{3 b})$ and $(\mathbf{3 c})$ had some interesting features. In compound (3b) ${ }^{1} \mathrm{H}$ NMR spectra has appeared NH proton as a broad singlet at $\delta=9.97 \mathrm{ppm}$, aromatic ring protons as a singlet at $\delta=7.66-7.80 \mathrm{ppm}, \mathrm{CH}$ protons as a doublet at $\delta=6.55 \mathrm{ppm}$ and $\mathrm{NH}_{2}$ protons as a singlet at $\delta=6.32 \mathrm{ppm}$. In compound $(3 \mathrm{c}){ }^{1} \mathrm{H}$ NMR spectra has appeared $\mathrm{NH}$ proton as a doublet at $\delta=7.96 \mathrm{ppm}$, aromatic ring protons as $\delta=6.6-7.30 \mathrm{ppm}$, $\mathrm{CH}$ proton as a doublet at $\delta=7.71 \mathrm{ppm}, \mathrm{NH}_{2}$ protons as a singlet at $\delta=6.06 \mathrm{ppm}$, two benzylic protons as a doublet at $\delta=4.47 \mathrm{ppm}$ and $\mathrm{OCH}_{3}$ protons as a singlet at $\delta=3.81 \mathrm{ppm}$.

The elemental analysis results of 9-substituted cyanopurines (4a-d) were satisfactory. The infrared spectrum of purines (4a-d) showed two strong absorptions for $\mathrm{CN}$ stretching vibrations in $2350-2380 \mathrm{~cm}^{-1}, \mathrm{C}=\mathrm{N}$ vibrations in $1510 \mathrm{~cm}^{-1}$ and $\mathrm{C}=\mathrm{C}$ stretching vibration in 1400-1475 $\mathrm{cm}^{-1}$. The mass spectrums showed a peak at $\mathrm{m} / z$ (\%) 265 (4a), 364 (4b), 293 (4c), 237 (4d) respectively.

The ${ }^{1} \mathrm{H}$ NMR spectra of compounds (4a) and (4b) were satisfactory. In compound (4a) ${ }^{1} \mathrm{H}$ NMR spectra has appeared $\mathrm{CH}$ proton of the pyrimidine as a singlet at $\delta=9.18 \mathrm{ppm}, \mathrm{CH}$ proton of the imidazole as a singlet at $\delta=9.09 \mathrm{ppm}$, aromatic ring protons as two doublet at $\delta=7.1-7.69 \mathrm{ppm}, \mathrm{CH}_{2}$ and $\mathrm{CH}_{3}$ protons as a quartet at $\delta=4.05 \mathrm{ppm}$ and a triplet at $\delta=1.31 \mathrm{ppm}$. In compound (4b) ${ }^{1} \mathrm{H}$ NMR spectra has appeared $\mathrm{CH}$ proton of the pyrimidine, $\mathrm{CH}$ proton of the imidazole, aromatic ring protons as a singlet at $\delta=9.41,9.2,8.19$ respectively.

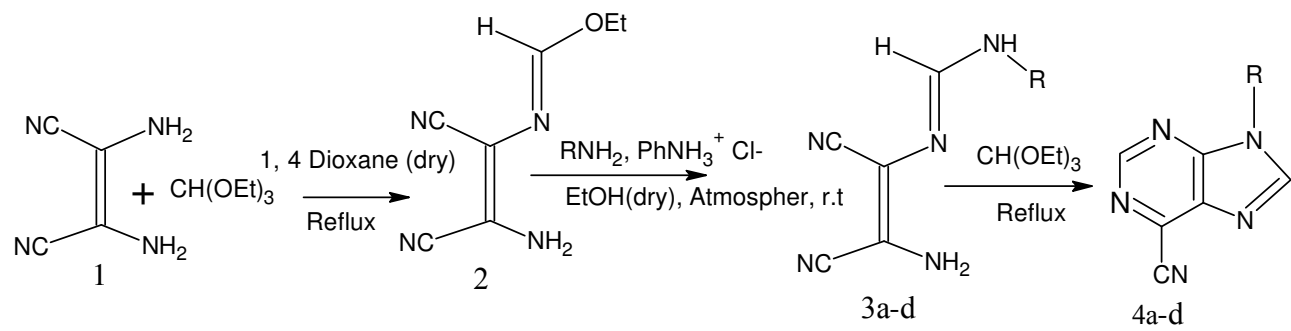

$a: R=4$-ethoxy aniline, $b: R=4$ amino aniline, $c: R=2$-methoxy benzyl amine, $d: R=4$-hydroxy animine

Scheme 1. Synthesis of novel 6-cyano-9(aryl)-9H-purine derivatives

\section{Conclusion}

In conclusion, a general and convenient synthesis of new 6-cyanopurine derivatives has been developed. The main advantages of these reactions are mild reaction conditions and high yields. The new compounds could be of interest in pharmacology and biology. 


\section{Acknowledgment}

The authors are grateful to the Guilan University and Islamic Azad University of Ghaemshahr for the financial support.

\section{References}

1. Booth B L, Alves M J and Proenca M F J R P, J Chem Soc Perkin Trans., 1990, 1, 1705.

2. Voet A B and Schwartz A W, Bioorg Chem., 1983, 12, 8.

3. Neilson D G, In the Chemistry of Amidines and Imidates, Wiley London, 1975, 1. 385-489.

4. Shriner R L and Neumann F W, Chem Rev., 1944, 35, 351-425.

5. Montgomery J A, Acc Chem Res., 1986, 19, 293.

6. Elion G B, Burg C and Hitchings G H, J Am Chem Soc., 1952, 74, 411.

7. Birkett P R, King H, Chapleo C B, Ewing D F and Mackenzie G, Tetrahedron, 1993, 49, 11029.

8. Matsumoto H, Hara S, Nagata N and Ikeda K, Heterocycl., 1995, 41, 47.

9. Robins R K, J Am Chem Soc., 1964, 7, 186.

10. Goldin A, Wood H B and Engle R R, Cancer Chemother Rep., 1968, 1, 1.

11. Montgomery J, Handb, Exp Pharmacol., 1974, 38, 76.

12. Henderson J F, Paterson A R P, Caldweel I C, Paul B, Chan M C and Lau K F, Cancer Chemother Rep., 1972, 3, 71.

13. Montgomery J A, J Med Res Rev., 1982, 2, 271.

14. MacKay L B and Hitchings G H, J Am Chem Soc., 1956, 78, 3511.

15. Hayashi E, Shimida N, Miyashita A and Yakugaku Z, Chem Abstr., 1977, 86, 121293.

16. Inoue H, Normura S and Ueda T, Nucleic Acids Symp Ser., 1981, 10, 19.

17. Yamane A, Matsuda M and Ueda T, Chem Pharm Bull., 1980, 28, 150.

18. Barlin G B and Young A C, J Chem Soc Perkin Trans., 1972, 1, 1269.

19. Giner-Sorolla A, Chem Ber., 1968, 101, 611.

20. Booth B L, Coster R D and Proenca M F, Synthesis, 1988, 8, 389.

21. Yahyazadeh A and Booth B L, Synth Commun., 2001, 31, 3225.

22. Yahyazadeh A, Russ J Org Chem., 2003, 39, 1718.

23. Yahyazadeh A and Sharifi Z, Phosphorus, Sulfur and Silicon and the related Elements, 2005, 17, 237-239.

24. Nami N, Neumuller B, Heravi M M and Haghdadi M, Mendeleev Commun., 2008, 18, 153.

25. Yahyazadeh A and Habibi F, E-J Chem., 2007, 4(3), 372-375.

26. Yahyazadeh A and Hossani F, E-J Chem., 2007, 4(3), 376-380.

27. Yahyazadeh A, Pourrostam B and Rabiee M, Bull Korean Chem Soc., 2003, 24, 1723. 


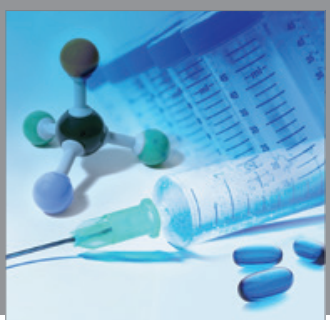

International Journal of

Medicinal Chemistry

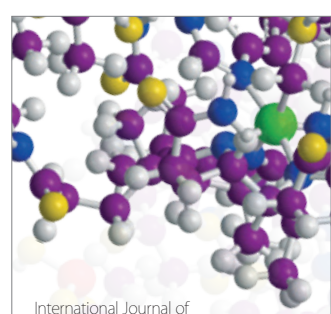

Carbohydrate Chemistry

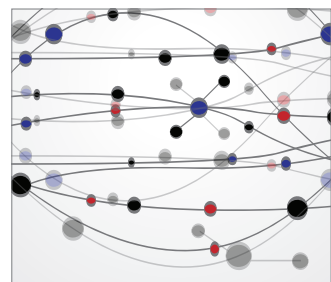

The Scientific World Journal
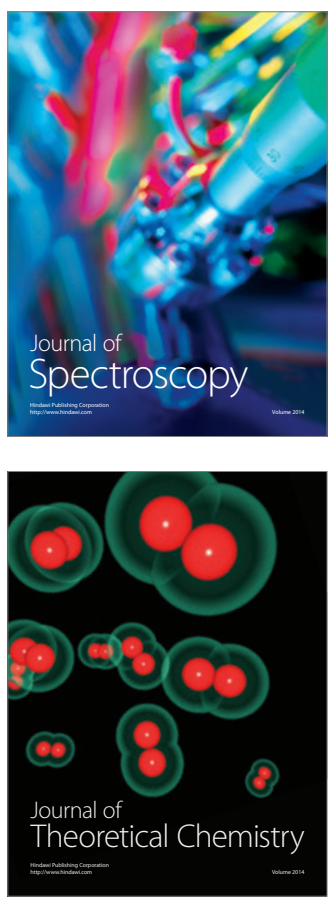
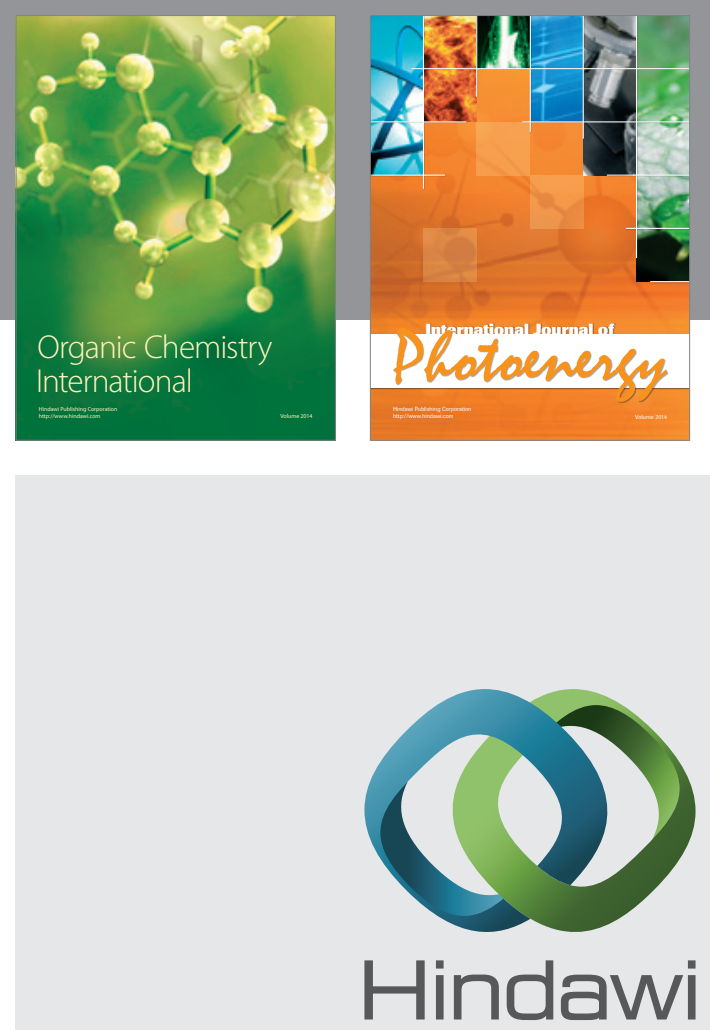

Submit your manuscripts at

http://www.hindawi.com
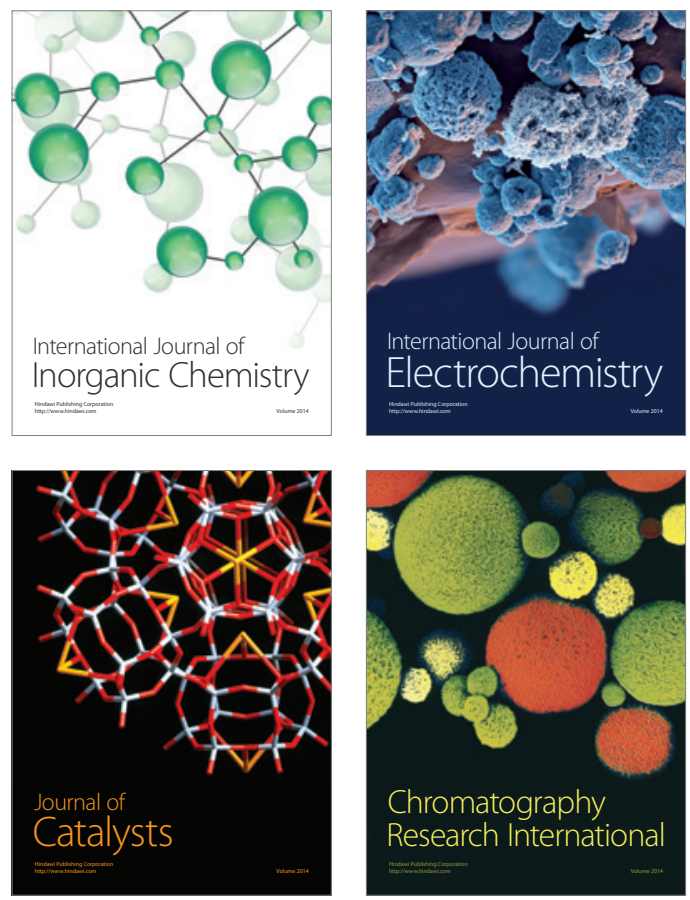
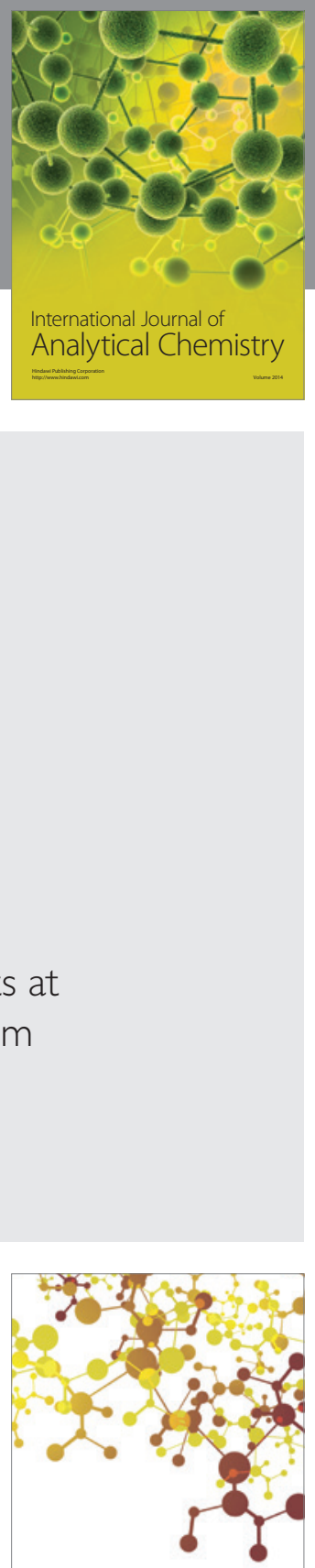

Journal of

Applied Chemistry
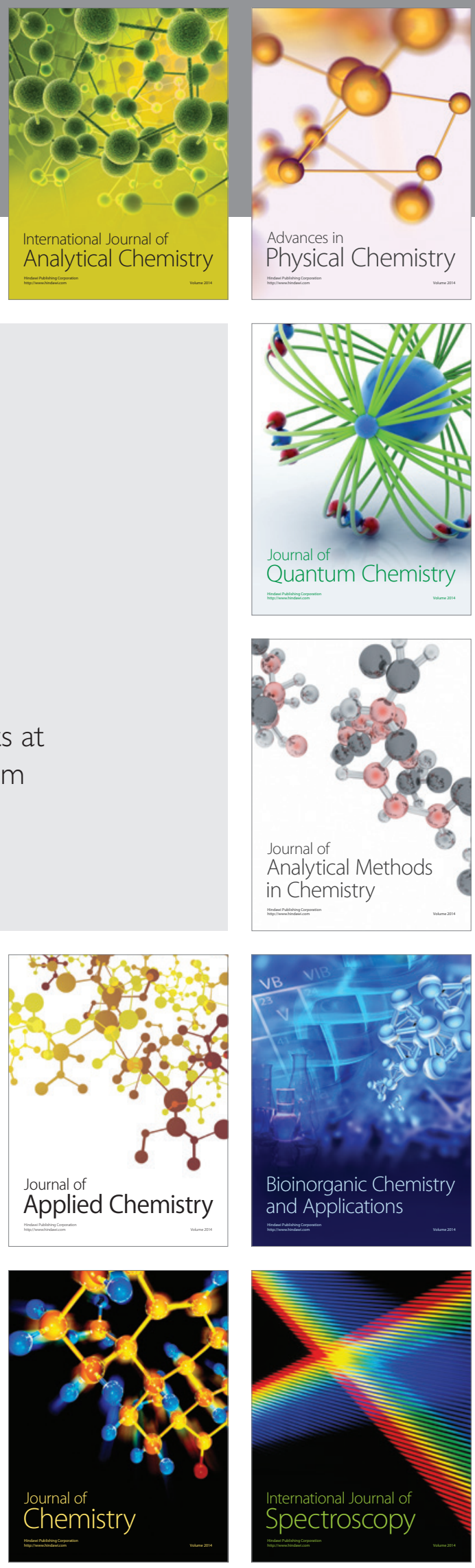